\title{
An Evidence-Based Review of Galcanezumab for the Treatment of Migraine
}

\author{
Ivan Urits · Melis Yilmaz $\cdot$ Karina Charipova · Kyle Gress • \\ Ehab Bahrum · Michael Swett · Amnon A. Berger · Hisham Kassem • \\ Anh L. Ngo · Elyse M. Cornett · Alan D. Kaye · Omar Viswanath
}

Received: July 30, 2020 / Accepted: September 15, 2020 / Published online: October 3, 2020

(C) The Author(s) 2020

\section{ABSTRACT}

Purpose of Review: This is a comprehensive review of the current literature on the usage of galcanezumab for migraine treatment. It reviews the biology, pathophysiology, epidemiology, diagnosis, and conventional treatment of migraines, then compares the literature available for galcanezumab with historical treatment options.

I. Urits $(\varangle)$ · A. A. Berger

Department of Anesthesiology, Critical Care, and Pain Medicine, Beth Israel Deaconess Medical Center, Harvard Medical School, Boston, MA, USA e-mail: iurits@bidmc.harvard.edu

M. Yilmaz · A. L. Ngo

Harvard Medical School, Boston, MA, USA

K. Charipova · K. Gress · E. Bahrum · M. Swett Georgetown University School of Medicine, Washington, DC, USA

H. Kassem

Department of Anesthesiology, Mount Sinai

Medical Center, Miami Beach, FL, USA

A. L. Ngo

Pain Specialty Group, Newington, NH, USA

E. M. Cornett

Department of Anesthesiology, Louisiana State University Health Sciences Center, New Orleans, LA, USA
Recent Findings: Migraine is a common headache disorder and constitutes a significant source of distress from both a personal and societal perspective. Conventional treatment includes abortive and preventive treatment. Treatment options are limited and may be only partially or minimally effective in some of the population. Recent evidence points to metabolic changes in the brain as possible causes of migraine, via reduced available energy or a spiking need for it, resulting in a relative insufficiency. This leads to trigeminocervical complex (TCC) activation and a headache

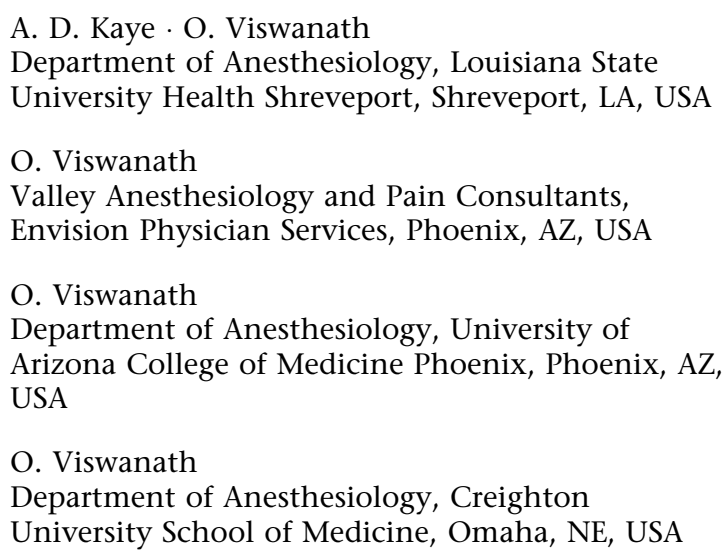
USA

O. Viswanath

Department of Anesthesiology, Creighton

University School of Medicine, Omaha, NE, USA 
episode, modulated by calcitonin gene-related peptide (CGRP). Galcanezumab (Emgality) is a monoclonal antibody targeting CGRP that is given in a monthly injection for the prevention of migraines. Its safety was previously shown in phase 1 and 2 trials, and recent phase 3 trials showed efficacy, with up to $50 \%$ reduction in monthly migraine days and improved functional capacity in migraineurs. Studies show that the drug is well tolerated and safe.

Summary: Migraine headache is a common neurological syndrome that causes great pain and suffering. Treatment options today are limited. Galcanezumab does not prevent migraines, but it is effective in decreasing their frequency and length. It is also much better tolerated than the currently existing therapies. While it is unlikely to provide monotherapy for migraines, it is a novel therapy that may be added for cases of severe or frequent migraines.

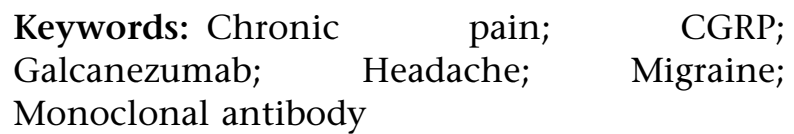

\section{Key Summary Points}

Migraine is a common headache disorder and constitutes a significant source of distress from both a personal and societal perspective. Treatment options are limited.

Recent evidence points to metabolic changes in the brain as possible causes of migraine, via reduced available energy or a spiking need for it, resulting in a relative insufficiency.

Galcanezumab (Emgality) is a monoclonal antibody, which targets CGRP, given in a monthly injection for the prevention of migraines.

While galcanezumab is unlikely to provide monotherapy for migraines, it is a novel therapy that may be added for cases of severe or frequent migraines.

\section{DIGITAL FEATURES}

This article is published with digital features to facilitate understanding of the article. To view digital features for this article, go to https://doi. org/10.6084/m9.figshare.12958013.

\section{INTRODUCTION AND EPIDEMIOLOGY}

Migraines are a frequent cause of neurological disorder among individuals in the United States. Migraine disorder disproportionately affects adult women, with $18 \%$ of women in the United States compared to $6 \%$ of men reporting migraines [1]. Data from the American Migraine Prevalence and Prevention (AMPP) Study examining epidemiologic factors such as racial and socioeconomic disparities demonstrated a higher prevalence in whites than in blacks and an inverse relationship between the prevalence of migraines and household income [2]. Data from the AMPP also supported an age disparity: the 30-39 age group is most affected by migraines across genders, with $7.4 \%$ of men and $24.4 \%$ of women in this age group experiencing migraine symptoms [2]. The prevalence of migraines has remained stable since 2005, as indicated by data from the National Health Interview Survey [3]. Migraines can be divided into a number of categories, including episodic migraine (15 or fewer migraine headaches per month) and chronic migraine (15 or more migraine headaches per month for greater than 3 months) [4, 5]. Sufferers of chronic migraine indicate a direct association of headache frequency with increased disease burden and reduced quality of life $[6,7]$. This disease burden has a negative effect on personal relationships as perceived by family members [6, 7]. Data from the Chronic Migraine Epidemiology and Outcomes (CaMEO) Study assessed familial burden with family members suffering from either chronic or episodic migraine [7]. These data included struggles within spousal relationships, perceived financial distress, and disease burden impact on children [7]. Given the existence of such a significant burden of disease, 
this review will examine traditional and explore novel treatment options for migraines.

This article is based on previously conducted studies and does not contain any studies with human participants or animals performed by any of the authors.

\section{RISK FACTORS}

A 2014 systematic review identified a number of associated triggers or risk factors predisposing individuals to migraines [8]. Ten precipitating factors for migraines were noted, in order of decreasing frequency: stress, auditory stimuli, fatigue, fasting, hormonal association with the female menstrual cycle, changes in sleep patterns, weather changes, visual stimuli, olfactory stimuli, and alcohol [8]. Stress was the most commonly reported trigger of migraines, with $58 \%$ of patients surveyed indicating stress as a trigger [8]. Alcohol was the least frequent trigger, with $27 \%$ of patients reporting it as a provoking factor [8]. A 2019 review suggested that an association between the aforementioned risk factors and metabolic disturbances may predispose individuals to migraine headaches [9]. The review emphasized a possible link between a brain experiencing a metabolic energy deficit and/or increased and overwhelming oxidative stress levels with migraine onset; the above scenarios may trigger a migraine response in order to return the brain to homeostatic function, implicating all risk factors associated with changes in brain metabolism and oxidative stress [9]. Along with these environmental triggers, genetics also play a role [10]. Genetic risk factors for migraine can be monogenic such as familial hemiplegic migraine (FHM), implicating the three genes CACNA1A, ATP1A2, and $S C N 1 A$, which predispose individuals to a rare hemiplegic type of migraine with aura [10]. The common form of migraine is associated with polygenic risk factors [10]. Multiple genomewide association studies (GWAS) have been performed on common migraine sufferers [10]. These GWAS have implicated numerous loci that may play a role in the pathophysiology of migraines and predispose individuals to migraines; further study is needed to elucidate the relevance of these loci [10].

\section{DIAGNOSIS AND CLINICAL PRESENTATION OF MIGRAINE}

The typical clinical presentation of a migraine consists of premonitory symptoms followed by a throbbing unilateral headache and subsequent postdromal symptoms $[11,12]$. Migraines may present with or without aura, a transient neurological disturbance [11]. Premonitory symptoms characterize the first phase of a migraine and may appear hours to days prior to the headache [12]. Yawning, fatigue, difficulty with mental concentration, and neck stiffness are common premonitory symptoms $[11,12]$. Given that the delineation between triggers and premonitory symptoms is not well defined, classic migraine triggers such as photophobia and osmophobia may likely be premonitory symptoms $[11,13]$. It is important to note that up to $72 \%$ of patients are able to accurately predict an impending migraine based on their premonitory symptoms [12]. Headache, head pain, and nausea characterize the second phase of a migraine, which may last for anywhere from hours to days [11]. Postdromal symptoms are diverse in presentation-the most common is simply fatigue-and characterize the third phase of migraines [11]. A migraine diagnosis can usually be derived from a complete history and physical evaluation alone, but additional testing such as magnetic resonance imaging (MRI) or computed tomography (CT) can be useful in ruling out other headache etiologies [14]. According to the AMPP, only $56.2 \%$ of patients suffering from migraine have received a formal medical diagnosis [15].

\section{PATHOPHYSIOLOGY}

The entire chronology of symptoms associated with migraines-the premonitory, headache, and postdromal phases-result from a complex brain network disorder associated with both environmental and genetic factors [4]. Current pathophysiology research identifies the 
trigeminovascular pathway as an important mechanism associated with the characteristic headache seen in migraines, as well as the distinct aura that is sometimes reported [4]. The trigeminovascular pathway comprises peripheral nociceptive axons originating from the meninges and vasculature $[4,16,17]$. The ophthalmic branch of the trigeminal nerve has been most frequently implicated in the symptomatology associated with migraines [4, 16, 17]. Research has shown that activation of the trigeminal nerves associated with the dura and vasculature are linked to general headache pain similar in quality to migraine [17]. These nociceptive axons release neuropeptides including calcitonin gene-related peptide (CGRP) into the trigeminocervical complex (TCC), a neural structure with many inputs that serves as a relay station before nociceptive information reaches higher cortical structures which elicit headache symptoms [4]. Signals from the TCC carry the nociceptive input from the trigeminal afferents to the thalamus, which plays a key role in central pain and sensory processing through connections to the cortex, including S1 and S2 of the somatosensory cortex $[4,16,17]$. The hypothalamus is intimately connected with various brain structures including the TCC and plays a role in pain processing $[4,16]$. Specifically, the premonitory symptoms are most directly linked to hypothalamic activation before the onset of headache symptoms, but research regarding premonitory symptom pathophysiology is lacking $[4,16,17]$. Many of the above pathways for migraine pathophysiology are described in novel research that has laid the foundation for the development of new treatment options [4].

\section{TRADITIONAL TREATMENT OPTIONS}

Individual treatment of migraine varies based on the patient and his or her symptomatology [15]. Patients suffering from migraine can treat their symptoms with over-the-counter (OTC) medications or prescription medications, or can decline treatment for acute symptoms [15]. These acute treatment options range from OTC nonsteroidal anti-inflammatory drugs (NSAIDs) to specific treatments targeting various pathways such as triptans or gepants [18]. The triptans target serotonin receptors while the gepants target the CGRP receptors in the brain [18]. Only $12 \%$ of patients opt to take preventive treatment for migraine [15]. Many of these patients use medications categorized as betablockers, calcium channel modulators, or anticonvulsants [18]. Many patients who undergo preventive treatment are less willing to continue preventive medications, as adherence is poor due to adverse events and low efficacy [19]. As the understanding of migraine pathophysiology evolves, novel treatment options will become increasingly available $[4,18]$. The gepant class provides an example of targeted therapy against the CGRP receptor via antagonism, which plays a role in the trigeminovascular pathway [18, 19]. Gepants offer better safety and tolerability compared to triptans [20]. Many other targeted pathways may play a key role in future migraine therapies, including glutamate receptors, notably the $N$-methyl-Daspartate (NMDA) receptor [18]. Recent progress in the acute treatment of migraines has moved toward monoclonal antibodies targeting pathophysiology pathways, notably the CGRP pathway [4, 20]. Monoclonal antibodies hold a distinct advantage in the treatment of migraine, because while many small-molecule drugs are prone to drug-drug interactions, monoclonal antibodies are engineered with specificity to a ligand or receptor, decreasing drug-drug interactions as well as unwanted adverse events [19]. Three monoclonal antibodies have been approved for the treatment of migraines in the United States: erenumab, fremanezumab, and galcanezumab [19].

\section{GALCANEZUMAB}

Galcanezumab, sold under the trade name Emgality, is a humanized monoclonal antibody developed by Eli Lilly and Company for the prevention of migraine in adults. Unlike like other preventive medications for migraines, which were initially designed to treat other conditions, galcanezumab was specifically 
developed for the treatment of migraines. It is a prescription taken once monthly that comes in a prefilled pen or syringe dosed at $120 \mathrm{mg}$. In September 2018, galcanezumab was approved in the United States for the prevention of migraine in adults [21]. More recently, Emgality $(300 \mathrm{mg}$ ) has been approved for the treatment of episodic cluster headaches. This monoclonal antibody targets the 37-amino-acid peptide calcitonin gene-related peptide (CGRP). The involvement of CGRP in nociception has also suggested the potential of galcanezumab in alleviating osteoarthritis-associated pain [22].

\section{MECHANISM OF ACTION}

Anti-calcitonin gene-related peptide monoclonal antibodies (anti-CGRP) are a new class of drugs directed against the potent vasodilator CGRP that is also involved in the transmission of nociception. CGRP is a neurotransmitter synthesized in the central and peripheral nervous system. It has an $\alpha$ and a $\beta$ form; however, there is not much known about the $\beta$ form [23]. Of note, the $\alpha$ form is most commonly found in the central and peripheral nervous system [23]. CGRP is synthesized in neuronal cell bodies and packaged into vesicles for transport to axonal terminals. When the presynaptic neuron containing CGRP is stimulated, CGRP is released from its storage vesicles in the axon terminal to the synaptic cleft in the process of calcium-dependent exocytosis. Following its release, CGRP binds to the CGRP receptor. The CGRP receptor is a protein complex composed of multiple proteins which are all required for the ligand's specificity to the receptor. The major components of the receptor include the G proteincoupled receptor calcitonin receptor-like receptor (CALCRL) and the receptor activity-modifying protein 1 (RAMP1) [23]. CALCRL has a $\mathrm{G}_{\alpha s}$ subunit which, when stimulated, activates the cAMP-dependent pathway, resulting in the production of cAMP. This process is mediated through stimulation of adenylate cyclase, which facilitates conversion of ATP to cAMP. cAMP acts as a second messenger activating protein kinase A (PKA), ultimately resulting in the phosphorylation of downstream targets. In the smooth muscle of cerebrovascular cells, the increase in cAMP due to CGRP results in vasodilation [23]. Although the pathophysiology of migraine is not completely understood, abnormal vasodilation has been shown to play an important role [24]. Given the above mechanism of action of CGRP, there are a few potential drug targets. The anti-CGRP galcanezumab works by binding the CGRP peptide with high affinity. This prevents CGRP from binding to its receptor, inhibiting a vasodilator response in cerebrovascular cells.

\section{PHARMACODYNAMICS AND PHARMACOKINETICS}

The analysis of pharmacodynamics and pharmacokinetics is critical in the development of drugs. Pharmacodynamics describes how the drug affects the body. It focuses on the relationship between the respective dose of a drug and the resulting desired activity, along with its side effects, therapeutic window, and duration of action. Pharmacokinetics describes how the body affects the drug. It focuses on absorption, distribution, metabolism, and excretion of a drug, to enable safe and effective therapeutic management in patients. In contrast to smallmolecule drugs, the pharmacodynamic and pharmacokinetic properties of antibody drugs are more complex. The binding of antibody drugs to soluble ligands characterizes its therapeutic effect. Therefore, the kinetics of both the ligand and antibody must be accounted for in determining the pharmacodynamic and pharmacokinetic properties of antibody drugs [25].

When an antibody drug is administered, the goal is for the antibody to bind to its respective soluble ligand, thereby reducing the concentration of free soluble ligand available to bind the ligand receptor. In theory, the efficacy of the antibody drug is determined by the magnitude and duration of the reduction in free soluble ligand [25]. Galcanezumab is a humanized monoclonal antibody that binds to the soluble ligand CGRP. Although measuring free ligand and total ligand (free + bound) concentrations would be ideal in the understanding of ligand inhibition, biological challenges impede these 
direct measurements [25]. Instead, galcanezumab-tolerant electrochemiluminescence assays measuring total CGRP concentration and enzyme-linked immunosorbent assays measuring serum galcanezumab concentrations are used. In this way, the interaction between CGRP and galcanezumab can be characterized by a pharmacokinetic (PK)/pharmacodynamic (PD) antibody-ligand interaction model. The details of this model can be found in other studies [6]. The PK/PD model suggests that the ratio of the zero-order production rate constant $\left(k_{\mathrm{in}}\right)$ and the first-order elimination rate constant $\left(k_{\text {out }}\right)$ are in equilibrium. This means that an increase in $\left(k_{\text {in }}\right)$ predicts an increase in free CGRP concentration. In serum, the elimination of free circulating CGRP is faster than galcanezumab-CGRP complex [25]. As a result, when CGRP is bound to galcanezumab, CGRP takes on the properties of galcanezumab, causing a temporary increase in total CGRP serum concentration. During a migraine attack, the free CGRP concentration is elevated. Therefore, the aim of using galcanezumab as a preventive treatment of chronic migraines is to have the appropriate galcanezumab concentration that will inhibit any spike in free CGRP during a migraine attack [25]. The PK/PD model suggests that a $300 \mathrm{mg}$ dose of galcanezumab causes a rapid decrease in free CGRP within a day of administration, followed by a slow return to pre-dose free CGRP concentration. The maximum reduction in free baseline CGRP was found to range from $39 \%$ when dosed at $5 \mathrm{mg}$ to $97 \%$ when dosed at $240 \mathrm{mg}$ and $300 \mathrm{mg}$ in a simulation study of the percent change in free CGRP with monthly administration of a range of doses of galcanezumab [25]. The findings indicated that galcanezumab displayed both time- and dose-dependent reduction in CGRP.

Most antibody drugs are administered intravenously (IV), subcutaneously (SC), or intramuscularly (IM) to avoid absorption in the gastrointestinal tract and subsequent first-pass metabolism in the liver. Galcanezumab can be administered by IV, SC, or IM. However, although its IV administration facilitates rapid delivery and complete bioavailability to the systemic circulation, it may not be convenient for patients to make hospital visits to receive medication. IM and SC preparations enable selfadministration of galcanezumab by patients for chronic dosing. The effectiveness of IM and SC administration is limited by the large dosing requirements and the subsequent pain at the injection site from the large volume [25]. This is overcome by taking multiple injections of smaller doses. For the treatment of chronic migraine, galcanezumab is started with a loading dose (LD) of $240 \mathrm{mg}$ followed by oncemonthly (QM) SC injection of $120 \mathrm{mg}$. Galcanezumab has a half-life of 27 days with bioavailability of $50-100 \%$ in SC or IM administration [25]. Once injected via SC or IM, galcanezumab predominantly enters the lymphatic system and then slowly drains into the systemic circulation, reaching a maximum serum antibody concentration 5 days post-administration. Because of its large molecular weight and polarity, galcanezumab cannot simply diffuse across a cell membrane. Its primary mechanism of extravasation is driven by the hydrostatic pressure gradients between blood and tissue in the process of convective transport. Following extravasation, the binding of galcanezumab to tissue proteins hinders its distribution. Similar to other monoclonal antibodies, the tight junctions comprising the vascular endothelium of the brain prevent galcanezumab from crossing the blood-brainbarrier (BBB) [25]. The majority of galcanezumab is eliminated via intracellular catabolism by the liver, skin, muscle, and intestine. Once the antibody is broken down into smaller peptides, it can be taken up by surrounding proteins and macromolecules to undergo further enzymatic degradation.

\section{SAFETY AND EFFICACY}

\section{Overview}

In recent years, LY2951742 or galcanezumab, a calcitonin gene-related peptide (CGRP), has been studied for its effects on bone pain and migraines. While the mechanism of action is described above and has been a known vasodilator for decades, it has only recently been used therapeutically to decrease bone pain 
and migraines. Galcanezumab specifically binds to both the $\alpha$ - and $\beta$-CGRP receptors and has been studied extensively for safety, initially in nonhuman primates and then in human subjects [26]. Using the known physiological response of capsaicin-induced dermal blood flow reduction, testing galcanezumab's inhibitory effect of the CGRP pathway would theoretically lead to increased blood flow. This hypothesis was studied using Doppler-imaged blood flow as a biomarker that defined response to the CGRP receptor [26]. This initial proof-ofconcept phase 1 study showed that galcanezumab did potently inhibit the CGRP pathway in nonhuman primates, and subsequently in humans in clinical trials [26]. After this proof of concept, a phase 2 randomized double-blind study was established across 35 sites enrolling 218 patients to evaluate safety and initial efficacy response. A dose of $150 \mathrm{mg}$ galcanezumab versus placebo was injected once every 2 weeks for 12 weeks. This phase 2 study showed a significant reduction in headache days and also showed increases in mild side effects such as erythema around the injection site, upper respiratory tract infections, and less commonly abdominal pain [27]. Further safety testing was performed in a phase $2 b$ trial with 410 patients who were administered either 5 , 50,120 , or $300 \mathrm{mg}$ galcanezumab versus placebo once every 4 weeks for three cycles. No long-term severe adverse effects occurred in patients receiving either galcanezumab or the placebo. Transfusion-related injection-site reactions occurred more often in the galcanezumab group (13.9\%) than in the placebo group $(5.8 \%)$. This was true for pain around the injection site as well (11.4\% galcanezumab vs. $2.9 \%$ placebo). Upper respiratory tract infections and hypersensitivity events occurred at approximately the same rate in both groups$8-10 \%$ and $3-5 \%$, respectively [28]. A further double-blind phase $2 \mathrm{~b}$ study was performed to validate proof of concept in humans. Using the Migraine-Specific Quality of Life Questionnaire version 2.1 (MSQv2.1) and the Headache Impact Test (HIT)- 6 to determine outcomes, the study included 187 patients who received three monthly injections over 12 weeks, with the galcanezumab group showing statistically significant functional improvements independent of migraine headache days [29].

\section{REGAIN INITIAL 3-MONTH STUDY AND 9-MONTH OPEN-LABEL EXTENSION}

Galcanezumab then moved into phase 3 trials, and a number of study groups concurrently evaluated the drug's effectiveness in migraine. The REGAIN (Evaluation of Galcanezumab in the Prevention of Chronic Migraine) study group screened 1903 subjects and enrolled 1113 subjects, and over $90 \%$ of the enrolled subjects completed the treatment period. Inclusion criteria included age of 18-65 years and 4-14 migraine headache days a month. The study ran for 3 months, with a 9-month open-label extension. The subjects first underwent a medication washout period of at least 30 days and up to 4 months in the case of onabotulinumtoxin. The length of this washout period was drug-dependent. The subjects were then randomized to placebo group versus $120 \mathrm{mg}$ galcanezumab injection versus $240 \mathrm{mg}$ galcanezumab injection. At the 3-month endpoint, both the $120 \mathrm{mg}$ and $240 \mathrm{mg}$ doses showed a statistically significant decrease in monthly headache days versus placebo. The dose itself showed no meaningful difference between the two drug subgroups. As previously observed, the drug group did show increased injection site reactions, erythema, pruritus, and sinusitis, but overall showed no severe adverse reactions. In conclusion, the REGAIN study group found Class I evidence that galcanezumab is superior to placebo in reducing the number of monthly migraine headache days in patients with chronic episodic migraines [30].

When looking at extended outcomes in the open-label extension, migraine headache days continued to decrease, and the drug was effective in a higher number of patients. There was also no significant increase in reported adverse events [31].

Subgroup analyses were performed on the same REGAIN study cohort to divide patients into those who had failed $\geq 2, \geq 1$, and 0 
previous preventive interventions. Statistically significant reductions in monthly migraine headache days were found in all three groups, regardless of the presence or lack of prior failures. Further, significant functional improvements quantified by the MSQv2.1 were noted alongside a decrease in acute abortive migraine medication use [32].

\section{EVOLVE-1 AND EVOLVE-2 TRIALS}

Concurrently with the REGAIN trials, the EVOLVE (Evaluation of LY2951742 in the Prevention of Episodic Migraine) study group also conducted a phase 3 trial under the same conditions as above: a drug washout period followed by double-blind $120 \mathrm{mg}$ versus $240 \mathrm{mg}$ galcanezumab versus placebo, with the same inclusion criteria of a 1-year history of migraine and 4-14 migraine headache days per month in the same 2016-2017 time frame. In this case, the timing was slightly different, with the treatment group observed for 6 months followed by cessation of use and a 4-month followup. The EVOLVE-1 study looked at 858 subjects across 90 sites in North America. Of note, 83.7\% (718) of the study group were women. Similar results were found, in that galcanezumab significantly reduced monthly headache days by 4.7 and 4.6 days versus 2.8 days with placebo. Again, the effect of galcanezumab dosage was not meaningful. Adverse effects occurred in less than 5\% [33].

The EVOLVE-2 study looked at 915 subjects at 109 study sites around the world including sites in North America, Europe, the Middle East, and Asia. It followed the same methods and timeline as REGAIN and EVOLVE-1 and similarly found a significant decrease in headache days in those using galcanezumab versus placebo. EVOLVE-2 also found no significant difference between galcanezumab dosages or prevalent adverse effects [34]. Both EVOLVE-1 and EVOLVE-2 provide further Class I evidence for the use of galcanezumab for migraine reduction.

The EVOLVE- 1 and 2 trials also looked at the patient populations for the 4 months after the study was over and treatment had been discontinued. In EVOLVE-1, overall efficacy in the $120 \mathrm{mg}$ galcanezumab group decreased from a reduction of 5.2 migraine days per month at month 6 , the last month the drug was still being administered, to 4.1 days at month 10,4 months after the drug had been stopped. Similarly, in the $240 \mathrm{mg}$ galcanezumab group, the efficacy decreased from a reduction of 5.3 days at month 6 to 3.8 days at month 10 . Despite this, these levels still demonstrated a statistically significant improvement compared with placebo, which showed a reduction from baseline of 3.4 days at month 6 and 3.3 days at month 10. EVOLVE-2 showed similar results, with both the $120 \mathrm{mg}$ and $240 \mathrm{mg}$ galcanezumab groups demonstrating a statistically significant long-term reduction at 10 months compared with the placebo group. This statistically significant change between the previously treated galcanezumab group and the placebo group suggests potential long-term effects even after drug cessation and warrants further study [35].

The data from EVOLVE-1 and EVOLVE-2 were then pooled to perform subgroup analysis on patients with low-frequency episodic migraines-defined as 4-7 monthly migraine headache days-against patients with high-frequency episodic migraines-defined as 8-14 monthly migraine headache days. Admittedly, there were some limitations with regard to the population, which was $75 \%$ white and $85 \%$ female. Additionally, $66 \%$ of the patients had high-frequency migraines versus only $34 \%$ having low-frequency migraines. Interestingly, the mean time since diagnosis with either type of migraine was 20.3 years. In both cases, similar results were found, as galcanezumab reduced migraine headache days, and the dose effects of $120 \mathrm{mg}$ versus $240 \mathrm{mg}$ were not significant. In patients with high-frequency episodic migraine, galcanezumab reduced migraines by 5.5 days, as opposed to 2.5 days in patients with low-frequency episodic migraines. Given that the average initial number of migraine headache days was 10.82 for high-frequency and 5.79 for low-frequency, the drug reduced overall headache days in both populations by $45-50 \%$ [36]. 


\section{COMBINED REGAIN AND EVOLVE- $1 / 2$}

The above studies were recently pooled in a meta-analysis that arrived at the same conclusion that the studies repeatedly found, that regardless of dose, galcanezumab statistically decreases the number of migraine headache days (MHDs) in patients with episodic migraines [37]. The REGAIN and EVOLVE-1/2 studies were also pooled by a separate study group to look at initial non-responders who continued to receive galcanezumab, to determine whether a certain population had a delayed response to the medication. This group examined patients who, after 1 month, did and did not have a protocol-defined response in migraine reduction $(>50 \%$ reduction in MHDs). They further separated the non-responders into four groups of modest responders (30-50\% reduction), limited responders (10-30\% reduction), minimal/non-responders (10\% reduction to $10 \%$ increase in MHDs), and worsening ( $>10 \%$ increase in MHDs). In the cohort of 450 non-responders after 1 month (51.3\% of total subjects), the moderate responders were more likely than the limited responders to eventually achieve a protocol-defined reduction, and likewise the limited responders were more likely than the minimal responders. Of the modest responders, $61.9 \%$ crossed the $50 \%$ threshold during the study and were therefore deemed responders. This number declined to $42.6 \%$ for the limited responders, and only $34.1 \%$ and $19.6 \%$ of the minimal and worsening categories exceeded the threshold, respectively. These numbers were rerun after 2 months, and 290 subjects were still non-responders $(33 \%)$. At this point, only $50 \%$ of the modest, $40.8 \%$ of the limited, $17.9 \%$ of the minimal, and $9.3 \%$ of the worsening categories would reach $>50 \%$ reduction by the end of the 6-month study duration. Therefore, in general, a more immediate response provides a more positive outcome; however, even if galcanezumab provides initial worsening of symptoms in the first month, there is still evidence showing a $20 \%$ chance that symptoms can be reduced by more than 50\% through 6 months of treatment [38].

\section{Adverse Effects}

Migraine is associated with significant headache-related disability and impairment of daily living and functioning, often calculated using a scoring system for disability-adjusted life-years (DALY) [39]. Traditionally, treatments for migraine have been evaluated using monthly migraine headache days as a primary endpoint, but this simple quantitative approach disregards the effects of migraine on physical, emotional, and social aspects of life [39]. Thus, more recently, existing and potential treatments for migraine have started to be evaluated based on their ability to improve health-related quality of life (HRQoL) scores [39]. Given that most oral migraine medications must be up-titrated to an efficacious dose over the course of weeks, many patients experience reductions in HRQoL scores and increased disability secondary to premature discontinuation [39]. In order to assess the effects of galcanezumab not only on physical health but also on daily living and functioning, Ford and colleagues compared the MSQv2.1 and Migraine Disability Assessment (MIDAS) scores of patients with episodic migraine taking the drug versus placebo [39]. Data were reported from two randomized, double-blind, placebocontrolled phase 3 studies (EVOLVE-1 and EVOLVE-2) [39]. Patients were evaluated for 6 months according to MSQv2.1 and MIDAS assessments conducted at baseline and then either monthly (MSQv2.1) or at months 3 and 6 (MIDAS) [39]. Patients had baseline MSQv2.1 scores of less than $60 \%$, indicating considerable functional impairment due to migraine [39].

It is known that high measures of disability correlate with low measures of HRQoL [39]. Consequently, treatments that improve disability are associated with higher HRQoL scores [39]. Analysis by Ford and colleagues demonstrated that a 6-month trial of galcanezumab in patients with episodic migraine was associated with significant improvements in HRQoL measured by MSQv2.1 and significant reductions in disability measured by MIDAS [39]. With 
treatment, patients exceeded approximately $80 \%$ of the total possible score for the MSQv2.1, suggesting substantially improved functional capacity in daily life [39]. This improvement indicates significantly increased capabilities in work and daily activity, productivity, personal relationships, leisure, and emotional well-being [39]. More than $70 \%$ of the patients in the treatment group experienced a reduction of at least 50\% in MIDAS total score [39]. Patient improvement was similar across the two dose regimens (120 mg in EVOLVE-1 and $240 \mathrm{mg}$ in EVOLVE-2), and differences between galcanezumab and placebo were seen starting in the first month of treatment [39]. These results corroborated the findings of prior studies supporting an association between galcanezumab and reductions in migraine headache days, low discontinuation rates, and high safety and tolerability [39].

As mentioned earlier, safety and tolerability play an important role in determining patient adherence to treatment regimens. The adverse effect profile of galcanezumab was investigated in a subset of patients diagnosed with episodic or chronic migraine not previously exposed to the drug [40]. Patients were administered galcanezumab $120 \mathrm{mg}$ or $240 \mathrm{mg}$ subcutaneously once a month for a year [40]. Safety and tolerability were assessed by calculating the frequency of treatment-emergent adverse events (TEAEs), serious adverse events (SAEs), and adverse events (AEs) leading to discontinuation of treatment [40].

It was determined that approximately $78 \%$ of patients completed the trial, with $4 \%$ of patients experiencing an SAE and 5\% discontinuing the study as a result of AEs [40]. Treatment compliance exceeded $95 \%$ in patients who finished the trial [40]. The rate of discontinuation due to AEs was lower than the adverse-event-related rate of discontinuation with topiramate [40]. The TEAEs experienced most frequently were injection site discomfort, nasopharyngitis, upper respiratory tract infections, sinusitis, and back pain [40]. Although migraines are commonly thought to be associated with increased risk of suicidal ideation, no treatment-emergent suicidal behavior was reported in this study [40]. While one-third of patients experienced injection site AEs such as injection site pain or reaction, only five patients discontinued for this reason [40]. Injection site discomfort was mild to moderate in severity and occurred most often on the day of injection, with resolution on the following day [40]. None of the TEAEs, including injection site discomfort, were dose-dependent, with the exception of upper respiratory tract infections [40]. However, data from the phase 3 double-blind, placebo-controlled study showed that incidence of upper respiratory tract infections was similar across the $120 \mathrm{mg}, 240 \mathrm{mg}$, and placebo groups [40].

When the safety of galcanezumab was assessed by considering changes from baseline in vital signs, laboratory values, EKG parameters, and weight, there were no clinically meaningful differences between the two dose regimens and placebo [40]. Further, the study group, characterized by a mean of 10.6 migraine headache days per month at baseline, experienced reductions in migraine headache days of $5.6(120 \mathrm{mg})$ and 6.5 days (240 mg) [40]. This safety and effectiveness profile was consistent with those of two previous phase 2 and two phase 3 studies in patients with episodic migraines and one phase 3 study in patients with chronic migraines [40].

\section{Guidelines for Use}

Galcanezumab and other monoclonal antibodies acting on CGRP or its receptors (eptinezumab and fremanezumab) are new therapies for the prevention of episodic and chronic migraine [41]. The development of evidenceand expert-based guidelines for their use is still in progress [41]. The European Headache Federation (EHF) performed a systematic literature review to assess the quality of available evidence and to establish recommendations to guide clinicians in the use of the CGRP monoclonal antibodies [41]. EHF considered evidence from inception to November 2018, with 28 studies identified as eligible for consideration [41].

Analysis of the available data indicated that galcanezumab is effective for the prevention of migraine in patients with both episodic and 
chronic disease [41]. Phase 2 and 3 randomized controlled studies demonstrated that galcanezumab use decreased the number of migraine headache days, decreased the number of days of acute medication use, and improved disability [41]. Of note, galcanezumab was shown to be effective in chronic migraine patients with a long history of disease who had failed two or more preventive treatment regimens [41]. The effectiveness of galcanezumab in patients with severe episodic migraine and history of preventive treatment failure is not as well established and requires further investigation [41]. The aforementioned rapid onset of action of galcanezumab and its ease of use via subcutaneous injection with monthly or quarterly dosing increases convenience and patient adherence [41]. For this reason, the long-term efficacy, safety, and tolerability of the drug is also currently being evaluated in patients with episodic and chronic cluster headache (ClinicalTrials.gov Identifier NCT02797951) [42].

Data on the management of other oral prophylactic migraine treatments in association with galcanezumab are scarce. It is not believed that CGRP monoclonal antibodies interact with other migraine regimens to a large degree, and it is likely that these drugs can be used in combination [41]. The development of binding and/or neutralizing antibodies with galcanezumab use is infrequent and has not been shown to affect treatment [41]. Thus, it is fair to consider adding galcanezumab in patients with insufficient response to other therapies [41]. Likewise, it is reasonable to allow the use of additional preventive drugs when galcanezumab produces incomplete symptom relief [41]. At present, no information on the concurrent use of galcanezumab and onabotulinumtoxinA is available [41].

Given the demonstrated effectiveness and high patient adherence to galcanezumab, and the significant negative impact on quality of life associated with migraine, the EHF has concluded that it is reasonable to treat patients with galcanezumab and other CGRP monoclonal antibodies [41]. As stated, while drug-drug interaction with galcanezumab is unlikely, CGRP is the most potent known vasodilator peptide and is considered dangerous in patients with known disease of the vascular system [41]. While no evidence of increased cardiovascular events has been recorded to date, patients with cardiovascular and cerebrovascular disease have been excluded from existing clinical trials [41]. Thus, long-term safety studies with galcanezumab are needed to justify offering the drug to this subset of patients [41].

With respect to duration of treatment, the EHF found that it is reasonable not to stop treatment even in the absence of clinical response [41]. Given limited data on the sustainability of the effects of galcanezumab following withdrawal of treatment, it is also reasonable to continue the drug for at least 6-12 months in patients with positive response [41]. It is essential to recognize that the costs of galcanezumab are not yet entirely known, but are expected to be higher than other migraine drugs [41]. For this reason, while galcanezumab appears to be a suitable option for patients with contraindications to other prophylactic medications, comorbidities, or poor compliance, it is unlikely that this therapy will be accessible to all patients [41].

\section{CONCLUSION}

Migraines are a common neurological disorder, most common in women between the ages of 30 and 39 years. They are classified as either episodic or recurrent, depending on the frequency and duration of headache attacks. These severe headaches are often debilitating, causing disability and imposing a significant burden from a societal perspective, including loss of working days and productivity. Migraines cause a great deal of personal pain and suffering, impact families, and take their toll on society.

Migraines can be triggered by different events and stimuli, such as stress, auditory or visual stimuli, fatigue, changes in hormonal states, caloric intake, sleep patterns, and alcohol consumption. All of these triggers can influence the metabolic state of the brain, either by reducing the available energy the brain is able to use, or by increasing the required energy; both of these create a state of relative insufficiency. There is a genetic predisposition to 
migraines, and several studies have explored genomic risk factors.

A typical migraine consists of premonitory symptoms, a headache attack, and finally postdromal symptoms that are at times as severe as the headache itself. This phenomenon is driven by the likely physiology of TCC activation, generating a nociceptive signal that is then transported to the thalamus and interpreted as a pain attack.

Conventional migraine treatment includes NSAIDs, triptans, and gepants for abortive therapy, and migraine prophylaxis that is taken regularly by only $12 \%$ of those suffering from severe recurring attacks. The latter include betablockers, calcium channel modulators, and anticonvulsants, each carrying its own set of risks and side effects. Unfortunately, treatment is only partially successful in parts of the population and not effective at all in other parts. Novel treatment options are driven by recent research on targeting CGRP, perturbing the generation and propagation of the pain signal. Another novel approach involves the use of monoclonal antibodies, such as galcanezumab, driven by recent advances in migraine research.

Galcanezumab (Emgality) is a monoclonal antibody against CGRP that is dosed as a $120 \mathrm{mg}$ self-administered monthly injection for the prevention of migraines and was specifically designed for that purpose. Galcanezumab prevents the signaling cascade driven by CGRP and reduces the frequency of migraines. Its safety was initially shown in phase 1 and 2 studies. The phase 3 REGAIN trial was then able to show a significant decrease in migraine days and improvement in functionality in the intervention group, with no serious adverse events. Injection site side effects and sinusitis were the most common minor adverse events. Subgroup analysis showed that treatment was successful even in patients who had failed numerous previous lines of treatment.

Similar results were demonstrated by the EVOLVE-1 and 2 trials. These studies also showed a decrease in efficacy with interval after treatment was stopped; however, some efficacy was maintained for months following the trials. Interestingly, examination of the data for the groups of non-responding patients revealed good evidence to support continued treatment, with a likely delayed response in these patients.

The safety of galcanezumab is generally good, with $>95 \%$ compliance to treatment and only $4 \%$ of patients experiencing any side effects. The drug was deemed safe and highly tolerable and provides a good option in light of the lack of efficacy with conventional treatment. It is also much better tolerated than existing options. Phase 4 trials and post-marketing data will be needed to assess the longterm effects and efficacy of this drug.

Migraines are common and debilitating disorder. Their treatment is unlikely to involve one single drug, but more likely will involve avoidance of triggers, good prophylactic and abortive care, and interventional treatments in carefully selected patients. In the current void, galcanezumab is a good wrench in the toolbox for a physician with a migraineur for a patient.

Papers of particular interest, published recently have been highlighted as:

- Of importance: 9, 21

9. A review of current literature emphasizing the metabolic pathophysiology of migraines and the treatments that currently target it.

21. An animal model for galcanezumab development, proving its vascular actions and providing basic data to support the current pharmacological model.

- Of major importance: $23,25,34,35$

28. A multinational phase $2 b$ study of galcanezumab in patients with episodic migraines providing evidence of treatment safety and supporting the establishment of a longer phase 3 trial.

29. A phase $2 \mathrm{~b}$ study for the efficacy of galcanezumab providing evidence of its efficacy over 12 weeks and supporting a phase 3 trial.

39. Report of the EVOLVE-1 and EVOLVE-2 phase 3 clinical trials, providing evidence not only of reduction in migraine days in patients, but also significant increase in functionality and reduction in disability in these 
patients compared with placebo (but not other prophylactic care).

40. A long multinational phase 3 trial providing evidence to support a reduction of migraine days in a large population with the current model of selfadministered injections.

\section{ACKNOWLEDGEMENTS}

Funding. No funding or sponsorship was received for this study or publication of this article.

Authorship. All named authors meet the International Committee of Medical Journal Editors (ICMJE) criteria for authorship for this article, take responsibility for the integrity of the work as a whole, and have given their approval for this version to be published.

Disclosures. None of the authors (Ivan Urits, Melis Yilmaz, Karina Charipova, Kyle Gress, Ehab Bahrum, Michael Swett, Amnon A. Berger, Hisham Kassem, Anh L. Ngo, Elyse M. Cornett, Alan D. Kaye, and Omar Viswanath) have anything to disclose.

Compliance with Ethics Guidelines. This article is based on previously conducted studies and does not contain any studies with human participants or animals performed by any of the authors.

Data Availability. Data sharing is not applicable to this article, as no data sets were generated or analyzed during the current study.

Open Access. This article is licensed under a Creative Commons Attribution-NonCommercial 4.0 International License, which permits any non-commercial use, sharing, adaptation, distribution and reproduction in any medium or format, as long as you give appropriate credit to the original author(s) and the source, provide a link to the Creative Commons licence, and indicate if changes were made. The images or other third party material in this article are included in the article's Creative Commons licence, unless indicated otherwise in a credit line to the material. If material is not included in the article's Creative Commons licence and your intended use is not permitted by statutory regulation or exceeds the permitted use, you will need to obtain permission directly from the copyright holder. To view a copy of this licence, visit http://creativecommons.org/licenses/by$\mathrm{nc} / 4.0 /$.

\section{REFERENCES}

1. Burch RC, Buse DC, Lipton RB. Migraine: epidemiology, burden, and comorbidity. Neurol Clin. 2019;37(4):631-49.

2. Lipton R, Bigal M, Diamond M, Freitag F, Reed M, Stewart W, AA Group. Migraine prevalence, disease burden, and the need for preventive therapy. Neurology. 2007;68(5):343.

3. Burch R, Rizzoli P, Loder E. The prevalence and impact of migraine and severe headache in the United States: figures and trends from government health studies. Headache J Head Face Pain. 2018;58(4):496-505.

4. Ong JJY, Wei DYT, Goadsby PJ. Recent advances in pharmacotherapy for migraine prevention: from pathophysiology to new drugs. Drugs. 2018;78(4): 411-37.

5. Headache Classification Committee of the International Headache Society (IHS). The International Classification of Headache Disorders, 3rd edition. Cephalalgia. 2018;38(1):1-211.

6. Adams AM, Serrano D, Buse DC, Reed ML, Marske V, Fanning KM, Lipton RB. The impact of chronic migraine: the Chronic Migraine Epidemiology and Outcomes (CaMEO) Study methods and baseline results. Cephalalgia. 2015;35(7):563-78.

7. Buse DC, Scher AI, Dodick DW, Reed ML, Fanning KM, Manack Adams A, Lipton RB. Impact of migraine on the family: perspectives of people with migraine and their spouse/domestic partner in the CaMEO study. Mayo Clin Proc. 2016;91(5): 596-611.

8. Peroutka SJ. What turns on a migraine? A systematic review of migraine precipitating factors. Curr Pain Headache Rep. 2014;18(10):453-8. 
9. Gross EC, Lisicki M, Fischer D, Sándor PS, Schoenen $\mathrm{J}$. The metabolic face of migraine-from pathophysiology to treatment. Nat Rev Neurol. 2019;15(11):627-43.

10. Tolner EA, Houben T, Terwindt GM, De Vries B, Ferrari MD, Van Den Maagdenberg AMJM. From migraine genes to mechanisms. Pain. 2015;156(4): S64-74.

11. Karsan N, Goadsby PJ. Biological insights from the premonitory symptoms of migraine. Nat Rev Neurol. 2018;14(12):699-710.

12. Giffin NJ, Ruggiero L, Lipton RB, Silberstein SD, Tvedskov JF, Olesen J, Altman J, Goadsby PJ, Macrae A. Premonitory symptoms in migraine: an electronic diary study. Neurology. 2003;60(6):935-40.

13. Schulte LH, Jürgens TP, May A. Photo-, osmo- and phonophobia in the premonitory phase of migraine: mistaking symptoms for triggers? J Headache Pain. 2015;16(1):1-5.

14. Evans RW. Diagnostic testing for migraine and other primary headaches. Neurol Clin. 2019;37(4): 707-25.

15. Diamond S, Bigal ME, Silberstein S, Loder E, Reed $\mathrm{M}$, Lipton RB. Patterns of diagnosis and acute and preventive treatment for migraine in the United States: results from the American Migraine Prevalence and Prevention Study. Headache. 2007;47(3): 355-63.

16. Goadsby PJ, Holland PR. An update: pathophysiology of migraine. Neurol Clin. 2019;37(4):651-71.

17. Goadsby PJ, Holland PR, Martins-Oliveira M, Hoffmann J, Schankin C, Akerman S. Pathophysiology of migraine: a disorder of sensory processing. Physiol Rev. 2017;97(2):553-622.

18. Puledda F, Messina R, Goadsby PJ. An update on migraine: current understanding and future directions. J Neurol. 2017;264(9):2031-9.

19. Ceriani CEJ, Wilhour DA, Silberstein SD. Novel medications for the treatment of migraine. Headache. 2019;59(9):1597-608.

20. Goadsby PJ. Bench to bedside advances in the 21st century for primary headache disorders: migraine treatments for migraine patients. Brain. 2016;139(10):2571-7.

21. Lupi C, Guerzoni S, Negro A, Benemei S. Oncemonthly galcanezumab for the prevention of migraine in adults: an evidence-based descriptive review and potential place in therapy. Vol. 15, Therapeutics and Clinical Risk Management. Dove Medical Press Ltd.; 2019. p. 557-69.
22. Benschop RJ, Collins EC, Darling RJ, Allan BW, Leung D, Conner EM, Nelson J, Gaynor B, Xu J, Wang XF, Lynch RA, Li B, McCarty D, Oskins JL, Lin C, Johnson KW, Chambers MG. Development of a novel antibody to calcitonin gene-related peptide for the treatment of osteoarthritis-related pain. Osteoarthr Cartil. 2014;22(4):578-85.

23. Edvinsson L. The CGRP pathway in migraine as a viable target for therapies. Headache. 2018;58: 33-47.

24. Ho TW, Edvinsson L, Goadsby PJ. CGRP and its receptors provide new insights into migraine pathophysiology. Nat Rev Neurol. 2010;6:573-82.

25. Kielbasa W, Helton DL. A new era for migraine: pharmacokinetic and pharmacodynamic insights into monoclonal antibodies with a focus on galcanezumab, an anti-CGRP antibody. Cephalalgia. 2019;39(10):1284-97.

26. Vermeersch S, Benschop RJ, Van Hecken A, Monteith D, Wroblewski VJ, Grayzel D, De Hoon J, Collins EC. Translational pharmacodynamics of calcitonin gene-related peptide monoclonal antibody LY2951742 in a capsaicin-induced dermal blood flow model. J Pharmacol Exp Ther. 2015;354(3):350-7.

27. Dodick DW, Goadsby PJ, Spierings ELH, Scherer JC, Sweeney SP, Grayzel DS. Safety and efficacy of LY2951742, a monoclonal antibody to calcitonin gene-related peptide, for the prevention of migraine: a phase 2, randomised, double-blind, placebo-controlled study. Lancet Neurol. 2014;13(9):885-92.

28. Oakes TMM, Skljarevski V, Zhang Q, Kielbasa W, Hodsdon ME, Detke HC, Camporeale A, Saper JR. Safety of galcanezumab in patients with episodic migraine: a randomized placebo-controlled doseranging Phase 2b study. Cephalalgia. 2018;38(6): 1015-25.

29. Ayer DW, Skljarevski V, Ford JH, Nyhuis AW, Lipton RB, Aurora SK. Measures of functioning in patients with episodic migraine: findings from a double-blind, randomized, placebo-controlled phase $2 \mathrm{~b}$ trial with galcanezumab. Headache. 2018;58(8):1225-35.

30. Detke HC, Goadsby PJ, Wang S, Friedman DI, Selzler KJ, Aurora SK. Galcanezumab in chronic migraine: the randomized, double-blind, placebocontrolled REGAIN study. Neurology. 2018;91(24): E2211-21.

31. Detke H, Pozo-Roisch P, Reuter U, Dolezil D, Li LQ, Wang S, Aurora SK. One-year treatment with galcanezumab in patients with chronic migraine: 
results from the open-label phase of the regain study. Neurology. 2019;92(15).

32. Ruff DD, Ford JH, Tockhorn-Heidenreich A, Sexson M, Govindan S, Pearlman EM, Wang SJ, Khan A, Aurora SK. Efficacy of galcanezumab in patients with chronic migraine and a history of preventive treatment failure. Cephalalgia. 2019;39(8):931-44.

33. Stauffer VL, Dodick DW, Zhang Q, Carter JN, Ailani J, Conley RR. Evaluation of galcanezumab for the prevention of episodic migraine. JAMA Neurol. 2018;75:1080-8.

34. Skljarevski V, Oakes TM, Zhang Q, Ferguson MB, Martinez J, Camporeale A, Johnson KW, Shan Q, Carter J, Schacht A, Goadsby PJ, Dodick DW. Effect of different doses of galcanezumab vs placebo for episodic migraine prevention a randomized clinical trial. JAMA Neurol. 2018;75(2):187-93.

35. Stauffer VL, Wang S, Voulgaropoulos M, Skljarevski V, Kovacik A, Aurora SK. Effect of galcanezumab following treatment cessation in patients with migraine: results from 2 randomized phase 3 trials. Headache. 2019;59(6):834-47.

36. Silberstein SD, Stauffer VL, Day KA, Lipsius S, Wilson MC. Galcanezumab in episodic migraine: subgroup analyses of efficacy by high versus low frequency of migraine headaches in phase 3 studies (EVOLVE-1 and EVOLVE-2). J Headache Pain. 2019;20(1):75.
37. Ren Z, Zhang H, Wang R, Yuan Q, Pan L, Chen C. The treatment efficacy of galcanezumab for migraine: a meta-analysis of randomized controlled trials. Clin Neurol Neurosurg. 2019;186(291): 105428.

38. Nichols R, Doty E, Sacco S, Ruff D, Pearlman E, Aurora SK. Analysis of initial nonresponders to galcanezumab in patients with episodic or chronic migraine: results from the EVOLVE-1, EVOLVE-2, and REGAIN randomized, double-blind, placebocontrolled studies. Headache. 2019;59(2):192-204.

39. Ford JH, Ayer DW, Zhang Q, Carter JN, Leroux E, Skljarevski V, Aurora SK, Tockhorn-Heidenreich A, Upton RB. Two randomized migraine studies of galcanezumab. Neurology. 2019;93(5):508-17.

40. Camporeale A, Kudrow D, Sides R, Wang S, Van Dycke A, Selzler KJ, Stauffer VL. A phase 3, longterm, open-label safety study of galcanezumab in patients with migraine. BMC Neurol. 2018;18(1): 188-200.

41. Sacco S, Bendtsen L, Ashina M, Reuter U, Terwindt G, Mitsikostas D-D, Martelletti P. European headache federation guideline on the use of monoclonal antibodies acting on the calcitonin gene related peptide or its receptor for migraine prevention. J Headache Pain. 2019;20(1):6-39.

42. ClinicalTrials.gov. A phase $3 \mathrm{~b}$ multicenter, singlearm, open-label safety study of LY2951742 (galcanezumab) in patients with episodic or chronic cluster headache; 2021. 